\title{
Características da gastrite crônica associada a Helicobacter pylori: aspectos topográficos, doenças associadas e correlação com o status cagA
}

\author{
Helicobacter pylori associated gastritis: topographical pattern, associated diseases \\ and correlation with cagA status
}

Mônica Maria Demas Álvares'; Marília Marino²; Celso Affonso de Oliveira²; Cláudia Castro Mendes³; Adriana de Cássia Ferreira Costa ${ }^{1}$; Juliana Guerra4; Dulciene Maria de Magalhães Queiroz M.M. ${ }^{4}$; Ana Margarida Miguel Ferreira Nogueira ${ }^{1}$

\section{unitermos}

Infecção por Helicobacter pylori

Gastrite crônica

Amostras cagA-positivas

Patogênese

\section{resumo}

Objetivos: Analisar o padrão topográfico da gastrite por $H$. pylori em relação ao status cagA e associação com úlcera duodenal (UD) e carcinoma gástrico precoce (CaGp). Material e método: Estudamos prospectivamente 160 biopsias endoscópicas e 40 peças de gastrectomia por $\mathrm{CaGp}$. Amostras do antro e do corpo na pequena e grande curvaturas, da incisura e do tumor, foram processadas rotineiramente para histologia com histoquímica para caracterização da metaplasia intestinal (MI). O Helicobacter pylori foi avaliado por histologia, imuno-histoquímica, cultura e reação em cadeia de polimerase (PCR) e o status cagA por PCR. A gastrite crônica (GC) foi classificada segundo o sistema Sydney. Realizou-se estudo topográfico em 130 pacientes, analisando os parâmetros de gastrite comparativamente entre as áreas e em relação ao status cagA. Resultados: Cento e vinte e um pacientes apresentaram GC, 24 UD e 14 eram normais. Detectaramse amostras cagA-positivas em 59 pacientes com GC, 17 com UD e 24 com CaGp. Todos os parâmetros de GC foram significativamente mais intensos nos pacientes infectados por amostras cagA-positivas, que se associavam ainda à presença de atrofia e MI. A Ml foi significativamente mais intensa na porção média da pequena curvatura antral do que nas demais regiões. Nos pacientes com GC e CaGp, infectados por amostras cagA-positivas, inflamação e atividade acometiam igualmente o antro e corpo distal, enquanto a atrofia e a MI predominavam no antro, particularmente na pequena curvatura do antro médio. Nos pacientes cagA-negativos a GC era predominantemente antral, e na UD a GC apresentava predominância antral, independentemente do status cagA. Conclusões: Nossos dados sugerem que o padrão de gastrite por $\mathrm{H}$. pylori se relaciona com fatores de virulência da bactéria. As lesões pré-neoplásicas são significativamente mais intensas na pequena curvatura do antro médio, onde surge a maioria dos carcinomas gástricos $(\mathrm{CaG})$. abstract

Aims: We aimed to investigate the topographical pattern of $\mathrm{H}$. pylori gastritis in relation to cagA status and association with duodenal ulcer (DU) and early gastric carcinoma (EGCa). Materials and method: We studied chronic gastritis (CG) in 160 patients submitted to upper endoscopy and in 40 patients with EGCa submitted to gastrectomy. Samples were taken from the antrum and corpus in the lesser and greater curvatures, incisura and from the tumour and were processed routinely for histology and histochemistry for intestinal metaplasia (IM) characterisation. H. pylori was evaluated by histology, immunohistochemistry, culture, and PCR; $\operatorname{cag} A$ status was evaluated by polymerase chain reaction (PCR). CG was analyzed according to the updated Sydney System. In 130 patients a topographic study was conducted by the comparative analysis of the gastritis parameters among the areas and according to cag $A$ status. Results: CG was observed in 121 patients, 24 had UD and 14 were normal. cagA-positive strains were detected in 59 patients with GC, $17 \mathrm{DU}$ and 24 EGCa. All gastritis parameters were significantly more intense in the cagA-positive group than in cagA-negative one. Infection by cagA-positive strains was associated with atrophy and IM. IM was significantly more intense in the middle portion of the lesser curvature in the antral mucosa. In CG or EGCa patients infected with cagA-positive strains, inflammation and activity compromised equally the antrum and distal corpus, whereas atrophy and IM were more frequently found in the antral mucosa. In DU, CG was antral-predominant irrespectively of cagA status. Conclusions: According to our data the topographical pattern of $\mathrm{H}$. pylori-associated gastritis seems to be associated with cagA status. Precancerous lesions were significantly more intense in the antral lesser curvature, where most gastric carcinomas arise. key words

Helicobacter pylori infection

Chronic gastritis

cagA-positive strains

Pathogenesis

1. Professoras da Faculdade de Medicina da Universidade Federal de Minas Cerais (FM/UFMG); Departamento de Anatomia Patológica e Medicina Legal.

2. Professores da Faculdade de Medicina da Universidade Federal de Minas Cerais (FM/UFMG); Departamento de Clínica Médica.

3. Professora da FM/UFMG; médica do Hospital das Clínicas da UFMG.

4. Professoras da FM/UFMG; Departamento de Propedêutica Complementar.

Trabalho realizado no Departamento de Anatomia Patológica e Medicina Legal da FM/UFMG

Apoio financeiro da Coordenação de Aperfeiçoamento de Pessoal de Nível Superior (CAPES) e do Conselho Nacional de Desenvolvimento Cientifico e Tecnológico (CNPq). 


\section{Introdução}

O Helicobacter pylori é o principal agente causal de gastrite crônica (GC) e a infecção desempenha um papel na patogênese da úlcera péptica e do carcinoma gástrico $(\mathrm{CaG})^{(1,4,7)}$. Pacientes com gastrite crônica atrófica multi-

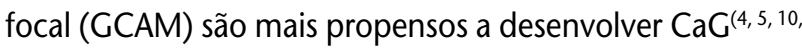
14,19,26), enquanto a úlcera duodenal (UD) está comumente associada à gastrite não-atrófica predominantemente antral(7). Diferentes padrões de gastrite associada a $H$. pylori podem ser determinados por fatores de virulência da bactéria, em associação com fatores do hospedeiro e

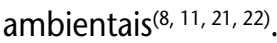

Vários estudos têm mostrado que a infecção por amostras cagA-positivas está associada a maior intensidade de inflamação, atividade, atrofia e metaplasia intestinal (MI) ${ }^{(10,}$ $19,21,24,29,31$ ). Apesar de relatos sobre a associação de infecção por amostras cagA-positivas com evoluções mais graves $(1,19$, 24,31), os elos de ligação entre o tipo de amostra infectante e o surgimento de lesões pré-cancerosas e $\mathrm{CaG}$ são ainda objeto de discussão(13).

O sistema Sydney atualizado recomenda a coleta de cinco biopsias: duas no antro e duas no corpo (em ambas as curvaturas) e uma na incisura ${ }^{(6)}$. Considera-se que esses locais sejam representativos de toda a mucosa gástrica, permitindo todos os diagnósticos possíveis. Entretanto, estudos sobre o padrão de gastrites predominantes do antro, do corpo e/ou do antro e corpo, bem como sobre a localização da atrofia e Ml, têm mostrado resultados controversos ${ }^{(4,15 \text {, }}$ 26,31). Além disso, poucos estudos enfocam a topografia na análise da GC por H. pylori ${ }^{(16,26,27,31)}$ e a avaliação de características topográficas da gastrite associada à infecção por amostras cagA-positivas tem sido pouco explorada.

Neste estudo pretendemos:

- analisar o padrão morfológico da gastrite associada a H. pylori em relação a dados demográficos dos pacientes, status cagA e associação com UD e carcinoma gástrico precoce (CaGp);

- investigar se a infecção por amostras cagA-positivas tem um padrão peculiar quando comparada à infecção por amostras cagA-negativas.

\section{Material e método}

Foram estudados prospectivamente 200 pacientes. Desses, 160 foram submetidos à endoscopia digestiva alta (EDA) para investigação de sintomas dispépticos e 40 apresentavam $\mathrm{CaGp}$. Houve o consentimento informado de todos os pacientes submetidos à EDA, os quais aceitaram participar da pesquisa. O estudo foi aprovado pelo comitê de ética da instituição.

\section{Biopsias endoscópicas}

Cem pacientes submeteram-se à EDA em hospitais da rede pública e 60 em serviço privado. Nenhum paciente usou qualquer medicamento por pelo menos 30 dias antes da realização da endoscopia, sendo excluídos aqueles submetidos previamente a cirurgias gástricas, portadores de distúrbios da coagulação, de neoplasias e/ou de qualquer doença grave.

Estudo topográfico da gastrite foi realizado em 130 pacientes com coleta de oito biopsias, segundo protocolo padronizado (Figura 1), na pequena e grande curvaturas do antro e corpo, incisura e parede anterior do corpo. Quatro biopsias adicionais do antro e do corpo foram colhidas para cultura e análise molecular de $\mathrm{H}$. pylori. Nos outros 30 pacientes coletaram-se pelo menos quatro biopsias para análise histológica, duas no antro e duas no corpo (pequena e grande curvaturas), e quatro biopsias para análise microbiológica.

\section{Peças cirúrgicas}

Dos arquivos do Serviço de Patologia Gastrintestinal foram selecionados 40 casos de $\mathrm{CaGp}$. Todas as peças cirúrgicas foram examinadas prospectivamente, de acordo com protocolo padronizado, com análise histológica de

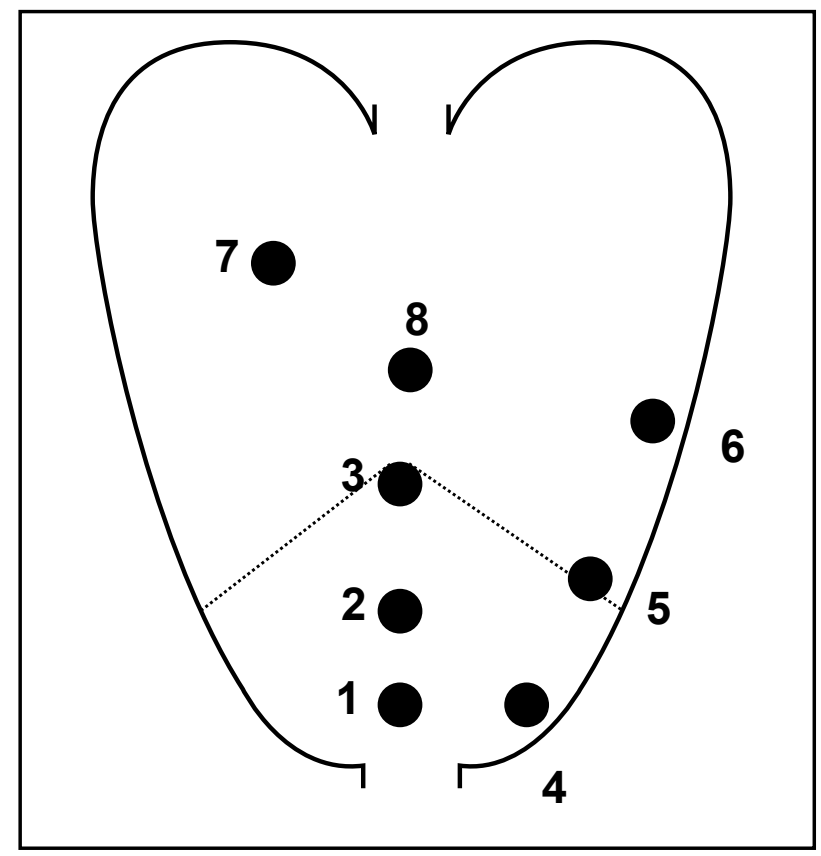

Figura 1 
todo o tumor, das mucosas gástricas de antro e de corpo nas duas curvaturas, da incisura e das margens de ressecção cirúrgica.

\section{Estudo histológico}

As amostras foram processadas rotineiramente para histologia:

- cortes histológicos de $4 \mu \mathrm{m}$ corados por hematoxilinaeosina para análise histológica;

- por carbolfucsina para detecção de $H$. pylori ${ }^{(25)}$;

- por PAS-Alcian Blue pH 2,5 e diamina férrica ${ }^{(30)}$ para caracterização da $\mathrm{Ml}^{(12)}$.

O H. pylori também foi pesquisado por imuno-histoquímica nos casos de $\mathrm{CaGp}$, segundo modificação do método do complexo avidina-biotina peroxidase $(A B C)^{(17)}$, usando-se kit comercial (Labvision) e anticorpo policlonal anti-H.pylori (Dakoppatts, USA) diluído a 1:100.

O diagnóstico histológico de GC foi realizado de acordo com o sistema Sydney, atualizado ${ }^{(6)}$ por duas observadoras, sem conhecimento prévio do status $H$. pylori e cagA. Os resultados discordantes foram examinados em conjunto pelas examinadoras e o resultado final desses casos foi o de consenso. Para o diagnóstico final de cada caso, considerou-se o maior grau de inflamação, atividade, atrofia e Ml. A presença de Ml e o seu tipo foram registrados em cada área e para cada caso. A GC foi classificada como não-atrófica e GCAM.

O padrão de gastrite foi classificado de acordo com o sistema Sydney em predominantemente antral ou predominante do corpo, se havia uma diferença de pelo menos dois graus na intensidade das variáveis quantitativas da gastrite, e em antro-corpo nas demais situações ${ }^{(6)}$. O diagnóstico de gastrite associada a $\mathrm{H}$. pylori foi firmado quando a bactéria foi detectada por pelo menos um dos métodos utilizados. Segundo Laúren, os $\mathrm{CaGp}$ foram classificados em intestinal, difuso e não-classificável(20).

Nos pacientes com GC associada a H. pylori a presença de atrofia, Ml e status cagA foi analisada em relação à procedência, gênero e idade dos pacientes. Os parâmetros histológicos e o padrão da gastrite foram analisados em relação aos status H. pylori e cagA.

\section{Estudo topográfico}

Para o estudo topográfico da gastrite por $H$. pylori os graus de inflamação, atividade, atrofia e Ml em cada uma das áreas examinadas foram comparados estatisticamente entre os grupos cagA-positivo e negativo e entre as áreas examinadas dentro de cada grupo H. pylori e cagA-positivo e negativo.

\section{Estudo microbiológico}

A cultura foi realizada em meio Belo Horizonte como descrito ${ }^{(24)}$. As amostras de mucosa gástrica, colhidas em $\mathrm{N} 2$ líquido e armazenadas a $-80^{\circ} \mathrm{C}$, bem como os isolados, foram testados para os genes ureA e cagA por PCR, como relatado ${ }^{(24)}$. Nos casos de $\mathrm{CaCp}$, as amostras das áreas mais representativas da gastrite por $\mathrm{H}$. pylori foram selecionadas e microdissecadas dos cortes histológicos, para a extração do DNA e identificação dos genes ureA e cagA pela técnica de PCR-nested (28).

O DNA foi extraído utilizando-se kit comercial (Nucleospin, BD Laboratories, Clontech). Os DNAs genômicos foram amplificados por PCR, utilizando-se conjuntos de primers descritos previamente ${ }^{(2,18,23)}$. A eletroforese foi realizada em gel de agarose a $1 \%$, contendo EDTA/tris/borato (GIBCO BRL, Gaithersburg, MD), corados com brometo de etídio e visualizados sob luz ultravioleta (UV) de comprimento de onda curto. Duas amostras cagA-positivas (ATCC 49503, NCTC 11.637) e uma cagA-negativa (TX $\left.30^{A}\right)$ foram usadas como controles positivo e negativo, respectivamente.

\section{Análise estatística}

Foram analisadas as diferenças de freqüência entre os grupos pelo teste $\mathrm{X}^{2}$ de duas caudas, com correção de Yates, ou pelo teste exato de Fisher. Também estimou-se a magnitude da associação entre infecção por amostras cagApositivas e atrofia, Ml e padrão da gastrite, em intervalos de confiança (IC) de $95 \%$, assim como foram cotejadas as idades dos pacientes pelo teste $t$ de Student. A intensidade da gastrite foi comparada entre os grupos pelo teste $U$ de Mann-Whitney e Kruskal-Wallis, e entre as áreas dentro do mesmo grupo pelo teste de Wilcoxon. O nível de significância foi estabelecido em $p<0,05$.

\section{Resultados}

Cento e vinte e um pacientes apresentaram GC; 24 , UD; um, úlcera péptica gástrica; 14 eram normais; e 170 estavam infectados por H. pylori (Tabela 1).

\section{Gastrite crônica}

Dos 121 pacientes com diagnóstico de GC, 107 estavam infectados por H. pylori: 59 por amostras cagA-positivas 
$(55,1 \%)$ e 48 por amostras cagA-negativas (44,9\%) (Tabela 2). Não houve diferença entre pacientes dos serviços público ou privado, nem em relação ao gênero dos pacientes quanto ao status cagA $(p=0,6 ; p=0,4)$, atrofia $(p=0,9$; $p=0,3)$ e MI $(p=0,2 ; p=0,8)$. Os pacientes com GCAM eram significativamente mais velhos ( $56 \pm 17$ anos) do que aqueles sem atrofia $(48,5 \pm 18,3$ anos: $p=0,04)$.

A MI não se relacionou com a idade dos pacientes, embora eles fossem mais velhos ( $57 \pm 15,4$ anos) do que os sem $\operatorname{Ml}(50,5 \pm 18,5$ anos: $p=0,09)$. Não houve diferença na idade dos pacientes quanto ao status cagA $(p=0,3)$.

Inflamação, atividade, atrofia e MI foram significativamente mais intensas nos pacientes $H$. pylori-positivos do que nos $H$. pylori-negativos, e naqueles infectados por amostras cagA-positivas em relação às negativas (Tabela 3).

A infecção por amostras cagA-positivas estava associada à presença de atrofia $(p=0,008)$ e $\mathrm{MI}(p=0,003)$, mas não houve associação com o tipo de MI (Tabela 4). A gastrite era de antro-corpo em 63 casos $(58,9 \%)$ e predominantemente antral em 44 casos (44,1\%), sem associação com o status cagA (Tabela 4).

\section{Úlcera duodenal}

Vinte e quatro pacientes com UD ( 16 homens e oito muIheres), média de idade de 50,4 $\pm 14,4$ anos, apresentavam GC associada a $H$. pylori, sendo que 17 (70,8\%) estavam infectados por amostras cagA-positivas. A inflamação e a atividade eram de intensidade moderada a acentuada, mas oito pacientes $(33,3 \%)$ apresentavam atrofia discreta com focos de MI do tipo I. Nove casos $(37,5 \%)$ tinham gastrite de antro-corpo e 15 (62,5\%) predominantemente antral.

\section{Carcinoma gástrico precoce}

Vinte e sete $(67,5 \%) \mathrm{CaCp}$ eram do tipo intestinal, 8 (20\%) do tipo difuso e $5(12,1 \%)$ eram não-classificáveis. Trinta e oito casos (95\%) eram H. pylori-positivos; em 34 casos o status cagA pôde ser determinado: 24 pacientes $(70,6 \%)$ estavam infectados por amostras cagA-positivas e $10(29,4 \%)$ por amostras cagA-negativas.

Trinta e sete $(92,5 \%)$ pacientes tinham GCAM associada a $H$. pylori, que era acentuada em $23(62,2 \%)$ e moderada em 8 (18,9\%); 34 casos (85\%) tinham Ml, acentuada

\section{Tabela 1 Grupos de diagnóstico e status H. pylori em 200 pacientes com gastrite crônica}

\begin{tabular}{lccc}
\hline \multirow{2}{*}{ Grupos diagnósticos } & \multicolumn{2}{c}{ Status H. pylori } & Total \\
Normal & H. pylori-positivo $(\%)$ & H. pylori-negativo (\%) & 14 \\
Gastrite crônica & 0 & $14(100)$ & 121 \\
Úlcera duodenal & $107(88,4)$ & $14(11,6)$ & 24 \\
Úlcera gástrica & $24(100)$ & 0 & 1 \\
Carcinoma precoce & $1(100)$ & 0 & 40 \\
Total & $38(95)$ & $2(5)$ & 200 \\
\hline
\end{tabular}

Tabela 2 e cagA

Características demográficas dos pacientes com gastrite crônica em relação ao status $H$. pylori

\begin{tabular}{|c|c|c|c|}
\hline & \multicolumn{2}{|c|}{ Sexo } & Idade \\
\hline & Masculino - $n(\%)$ & Feminino $-n(\%)$ & (anos $\pm d p)$ \\
\hline H. pylori+ cagA+ $(n=59)$ & $21(35,6)$ & $38(64,4)$ & $50,4 \pm 17,3$ \\
\hline H. pylori+ cagA- $(n=48)$ & $22(45,8)$ & $26(54,2)$ & $53,7 \pm 18,6$ \\
\hline H. pylori- $(n=14)$ & $9(64,3)$ & $5(35,7)$ & $45,7 \pm 17,4$ \\
\hline Total & 52 & 69 & $51,5 \pm 18$ \\
\hline
\end{tabular}

DP: desvio-padrão. 


\section{Tabela 3 Características da gastrite crônica em relação ao status H. pylori e cagA}

\begin{tabular}{|c|c|c|c|c|c|}
\hline \multirow[t]{2}{*}{ Parâmetros de gastrite } & \multirow{2}{*}{$\begin{array}{c}\text { H. pylori negativo } \\
n(\%)\end{array}$} & \multicolumn{2}{|c|}{ H. pylori positivo } & \multicolumn{2}{|c|}{ Valor de $p$} \\
\hline & & cagAt $(\%)$ & cagA- $(\%)$ & status H. pylori & status cagA \\
\hline Inflamação & & & & $p=0,0001$ & $p=0,01$ \\
\hline Ausente & 0 & 0 & 0 & & \\
\hline Discreta & $13(92,9)$ & $5(8,5)$ & $7(14,6)$ & & \\
\hline Moderada & $1(7,1)$ & $37(62,7)$ & $37(77,1)$ & & \\
\hline Acentuada & 0 & $17(28,8)$ & $4(8,3)$ & & \\
\hline Atividade & & & & $p=0,0001$ & $p=0,03$ \\
\hline Ausente & $8(57,1)$ & $1(1,7)$ & 0 & & \\
\hline Discreta & $6(42,9)$ & $11(18,6)$ & $15(31,3)$ & & \\
\hline Moderada & 0 & $24(40,7)$ & $24(50)$ & & \\
\hline Acentuada & 0 & $23(39)$ & $9(18,8)$ & & \\
\hline Atrofia & & & & $p=0,02$ & $p=0,002$ \\
\hline Ausente & $12(85,7)$ & $23(39)$ & $32(66,7)$ & & \\
\hline Discreta & $1(7,1)$ & $24(40,7)$ & $14(29,2)$ & & \\
\hline Moderada & $1(7,1)$ & $5(8,5)$ & $1(2,1)$ & & \\
\hline Acentuada & 0 & $7(11,9)$ & $1(2,1)$ & & \\
\hline Metaplasia intestinal & & & & $p=0,02$ & $p=0,002$ \\
\hline Ausente & $14(100)$ & $37(62,7)$ & $43(89,6)$ & & \\
\hline Discreta & 0 & $12(20,3)$ & $3(63)$ & & \\
\hline Moderada & 0 & $8(13,6)$ & $1(2,1)$ & & \\
\hline Acentuada & 0 & $2(3,4)$ & $1(2,1)$ & & \\
\hline
\end{tabular}

\section{Associação entre status cagA e atrofia, metaplasia intestinal e padrão de gastrite na infecção}

Tabela 4 por $H$. pylori de acordo com o sistema Sydney

\begin{tabular}{|c|c|c|c|c|c|}
\hline & $\operatorname{cagA}+$ & cagA- & OR & IC 95\% & Valor de $p$ \\
\hline Atrofia & & & 3,13 & $1,31-7,53$ & 0,008 \\
\hline Presente & $36(69,2 \%)$ & $16(30,8 \%)$ & & & \\
\hline Ausente & $23(41,8 \%)$ & $32(58,2 \%)$ & & & \\
\hline Metaplasia intestinal & & & 5,11 & $1,61-17,23$ & 0,003 \\
\hline Presente & $22(81,5 \%)$ & $5(18,5 \%)$ & & & \\
\hline Ausente & $37(46,3 \%)$ & $43(53,8 \%)$ & & & \\
\hline Metaplasia intestinal & & & 1,25 & $0,12-12,13$ & 1 \\
\hline Tipo incompleto & $15(78,9 \%)$ & $4(21,1 \%)$ & & & \\
\hline Tipo completo & $6(75 \%)$ & $2(25 \%)$ & & & \\
\hline Metaplasia intestinal & & & 2 & $0,15-55,39$ & 1 \\
\hline Tipo III & $6(85,7 \%)$ & $1(14,3 \%)$ & & & \\
\hline Tipos I e II & $15(75 \%)$ & $5(25 \%)$ & & & \\
\hline Padrão de gastrite & & & 1,08 & $0,48-2,62$ & 0,9 \\
\hline Predominante no antro & $25(56,8 \%)$ & $19(43,2 \%)$ & & & \\
\hline Antro/corpo & $34(54 \%)$ & $29(46 \%)$ & & & \\
\hline
\end{tabular}

OR: odds ratio; IC: intervalo de confiança. 
em 14 (41,2\%) e moderada em 11 (32,4\%). A MI era do tipo completo em $12(35,3 \%)$ e do incompleto em 22 $(64,7 \%)$, dos quais 16 eram do tipo III. A atividade era de igual intensidade no antro e no corpo em 18 casos (45\%) e predominava no corpo em 12 (30\%).

Atrofia e MI foram significativamente mais freqüentes e intensas no antro do que no corpo $(p=0,0001)$, cujas porções distais estavam mais comprometidas, e não se observou associação entre essas lesões e o status cagA ( $p \geq$ $0,7)$. No diagnóstico final o padrão de gastrite foi de antrocorpo em 27 casos (67,5\%), predominantemente antral em 11 (27,5\%) e predominante do corpo em 2 (5\%).

\section{Estudo topográfico}

Intensidade da inflamação, atividade, atrofia e Ml em cada uma das áreas examinadas nos pacientes com GC, associadas ao H. pylori, são apresentadas na Figura 2. A inflamação foi significativamente mais intensa em todas as áreas do antro e na incisura do que no corpo $(p<0,05)$. A atividade foi semelhante no antro, incisura e pequena curvatura do corpo e significativamente mais intensa que nas demais áreas do corpo. A atrofia foi mais intensa no antro (áreas 2 e 4) e corpo distal ao longo da pequena curvatura, poupando as porções mais distais do antro e proximais do corpo $(p<0,05)$. A MI foi significativamente mais intensa na pequena curvatura do antro médio do que em outras regiões da mucosa. $\mathrm{Na}$ GC H. pylori-negativa, inflamação e atividade foram predominantemente de intensidade discreta, sem diferença significativa entre as regiões.

$\mathrm{Na}$ Figura 3 pode-se comparar a intensidade dos parâmetros da GC em todas as áreas nos grupos infectados por amostras cagA-positivas e negativas. As diferenças entre esses

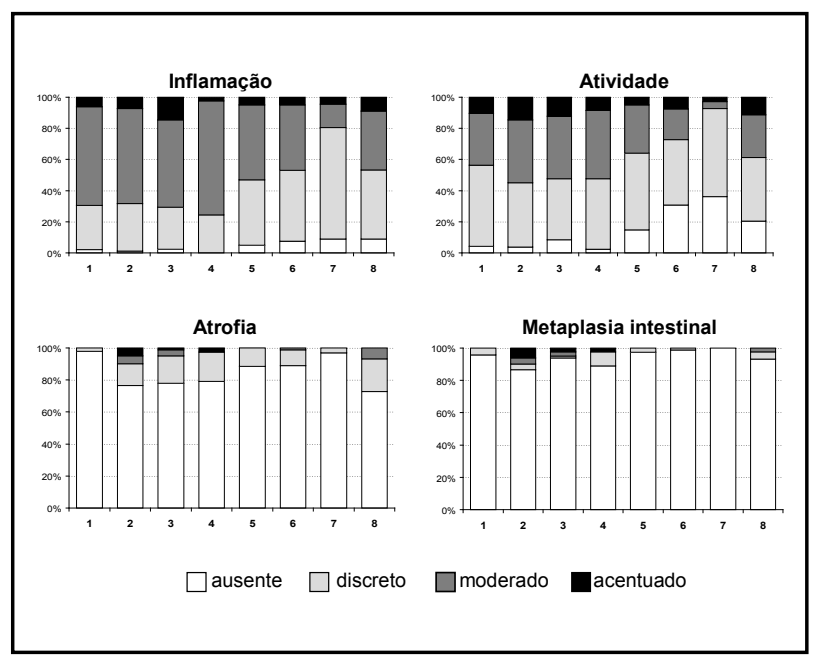

Figura 2

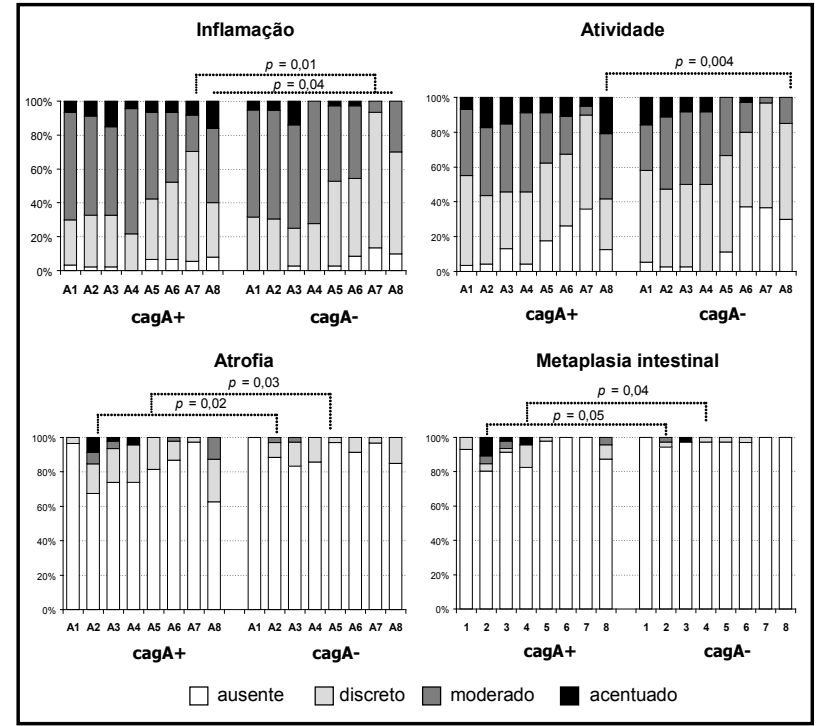

Figura 3

grupos foram significativas para todas as variáveis da GC, mas em áreas distintas. Os pacientes infectados por amostras cagA-positivas apresentavam inflamação e atividade mais intensas no corpo (pequena curvatura e parede anterior) ( $p$ $\leq 0,04)$ e atrofia e Ml mais intensas no antro $(p \leq 0,05)$ do que pacientes infectados por amostras cagA-negativas.

A intensidade dos parâmetros da GC foi em seguida comparada entre as áreas em cada grupo. Nos pacientes cagA-positivos o antro e a porção distal do corpo apresentavam o mesmo grau de inflamação e atrofia, enquanto nos cagA-negativos o antro estava significativamente mais comprometido do que o corpo $(p<0,05)$. Nos casos cagApositivos a atividade era mais intensa no antro e pequena curvatura do corpo e a Ml era significativamente mais intensa na porção média da pequena curvatura antral. Nessa região, a atrofia estava associada à infecção por amostras cagA-positivas (OR, 3,75; IC 95\%, 1 - 15,2; $p=0,049$ ). No grupo cagA-negativo, atrofia e MI foram menos freqüentes e sem diferença significativa entre as áreas.

Nos pacientes com UD, inflamação e atividade eram acentuadamente mais intensas no antro do que no corpo (inflamação $p \leq 0,02$; atividade $p<0,03$ ), sem diferença entre os diversos locais da mucosa antral $(p>0,4)$ e independente do status cagA. Atrofia e Ml focais foram observadas somente no antro.

\section{Discussão}

As características da GC associada à infecção por $H$. pylori têm sido amplamente estudadas $(7,10,14,15,16,19,21,26,27,31)$, mas alguns aspectos permanecem por esclarecer. 
No presente estudo a população estudada foi relativamente homogênea quanto ao status $H$. pylori e cagA e presença de atrofia e $\mathrm{Ml}$, que foram independentes da procedência ou gênero dos pacientes. Observamos apenas relação significativa entre a idade dos pacientes e a presença de atrofia, o que está de acordo com a evolução da $\mathrm{GC}^{(3)}$. Na GC associada a H. pylori, bem como nos pacientes infectados por amostras cagA-positivas, a intensidade da inflamação, atividade, atrofia e $\mathrm{Ml}$ foi significativamente maior que nos pacientes não-infectados e que naqueles infectados por amostras cagA-negativas, respectivamente. Além disso, amostras cagA-positivas estavam significativamente associadas à presença de atrofia e $\mathrm{Ml}$, demonstrando que a infecção por essas amostras se relaciona com evoluções mais graves ${ }^{(19)}$. Esses resultados são idênticos aos de outros estudos ${ }^{(10,19,21,29)}$.

Observamos freqüência semelhante de MI dos tipos II e III, tanto no grupo de GC como no de CaGp, sem relação com a infecção por amostras cagA-positivas, diferentemente dos resultados de outros autores ${ }^{(21)}$. Isso pode ser devido ao pequeno número de casos que estudamos e à baixa freqüência de atrofia e a Ml entre os casos cagA-negativos.

A gastrite é um processo dinâmico e diferentes etapas do mesmo processo podem ser analisadas e classificadas em diversas categorias. A utilização de protocolos diferentes implica muitas vezes em resultados diferentes, gerando controvérsias.

$\mathrm{Na}$ atualização do sistema Sydney foi estabelecido que, para classificar a GC como predominante do antro ou do corpo, deve haver uma diferença de dois graus na intensidade das variáveis ${ }^{(6)}$. Entretanto, não está claro se esse critério deve ser aplicado apenas para inflamação e atividade e se independe da presença de atrofia. De acordo com esses critérios, observamos predomínio de casos de gastrite antrocorpo nos pacientes com GC não-ulcerosa (59\%) e naqueles com CaGp (67,5\%), enquanto $62,5 \%$ dos pacientes com UD apresentavam gastrite predominantemente antral.

Os resultados estão de acordo com a classificação proposta por Correa ${ }^{(3)}$ e posteriormente incorporada ao sistema Sydney ${ }^{(6)}$, segundo a qual a GC por H. pylori predominantemente antral está relacionada à UD, enquanto a GC de antro e corpo é mais comumente observada nos casos de $\mathrm{CaG}$. Todavia, 37,5\% dos casos de UD associavam-se à gastrite de antro-corpo e 27,5\% dos casos de CaGp associavam-se a GC predominantemente antral. Além disso, tal como relatado por outros ${ }^{(31)}$, essa avaliação não permitiu discriminar o padrão de gastrite em relação à infecção por amostras cagA-positivas. O padrão de gastrite que determina a asso- ciação com UD e CaG resulta provavelmente da interação de fatores da bactéria $(1,10,22,24,26,31)$, do hospedeiro ${ }^{(4,8,11)}$ e da dieta ${ }^{(4)}$, cujas repercussões nem sempre são avaliáveis morfologicamente.

A análise topográfica nos permitiu perceber que na GC não-atrófica associada ao $\mathrm{H}$. pylori, independentemente da associação com UD, a inflamação e a atividade eram mais intensas no antro que no corpo, o que está de acordo com a literatura ${ }^{(14,26,27,29,31)}$. Porém, nos casos de GCAM associados ao $\mathrm{H}$. pylori, particularmente naqueles em que a atrofia e MI eram no mínimo de grau moderado, essas lesões eram mais intensas no antro, ao passo que a inflamação e atividade eram mais intensas na porção distal do corpo, em comparação com os casos sem atrofia. Esses casos foram diagnosticados como gastrite de antro e corpo, considerando a inflamação e atividade, embora a atrofia, lesão biologicamente mais significativa, fosse mais intensa no antro.

Poucos estudos enfocam a questão da topografia na análise da GC por $H$. pylori e, tanto quanto sabemos, não há estudos enfatizando a relação com amostras virulentas, objetivo do nosso trabalho.

Diferentemente do resultado obtido quando subtipamos a gastrite em predominantemente antral ou de antro e corpo, o estudo topográfico permitiu definir um padrão de gastrite relacionado com a infecção por amostras virulentas. Observamos que na GC associada a amostras cagA-positivas, antro e corpo distal estavam igualmente acometidos, independentemente da presença de atrofia. Nos casos de GCAM cagA-positivos, a inflamação e atividade foram significativamente mais intensas no corpo e a atrofia e MI foram mais intensas no antro, quando comparadas ao grupo cagA-negativo. Por outro lado, a inflamação e a atividade foram significativamente mais intensas no antro que no corpo e a atrofia e Ml foram pouco freqüentes no grupo cagA-negativo. Esses dados sugerem que a atrofia antral modula o grau de inflamação e atividade no corpo.

Admite-se que o antro atrófico com MI torna-se hostil ao microrganismo que passa a colonizar mais densamente o corpo, com conseqüente aumento da reação inflamatória ${ }^{(8)}$. Fatores do hospedeiro, como bloqueio da secreção ácida por interleucinas podem reforçar esse quadro(8). É interessante ressaltar que em nosso estudo a gastrite por amostras cagA-positivas foi distinta em pacientes ulcerosos e não-ulcerosos. Nos pacientes com UD a inflamação e atividade foram significativamente mais intensas no antro que no corpo. Provavelmente, fatores do hospedeiro, modulando a secreção ácida, são importantes na evolução do quadro e determinam a associação com UD. 
Demonstramos que a $\mathrm{Ml}$ foi mais freqüente e mais intensa na porção média do antro na pequena curvatura. A entidade GCAM proposta por Correa ${ }^{(3)}$ e adotada pelo sistema Sydney atualizado(6) considera que a atrofia e a $\mathrm{Ml}$ começam em torno da incisura e se estendem para o resto da mucosa antral, em direção ao piloro, ao longo da pequena curvatura e da transição antro-corpo. Esse padrão de gastrite, que serviu de base para estudos prospectivos do mesmo grupo ${ }^{(3,4)}$, foi descrito inicialmente em necropsias numa população colombiana ${ }^{(5)}$.

A despeito de parecer reprodutível em outras populações, há dúvidas se este é o único padrão de gastrite atrófica ${ }^{(15)}$. Além do mais, deve-se considerar que populações de risco aumentado para $\mathrm{CaC}$ são afetadas por múltiplos fatores agressivos, particularmente relacionados à dieta e infecção por H. pylori e por amostras cagA-positivas do microrganismo ${ }^{(4)}$.

Nossos resultados não sustentam a hipótese de que a GCAM começa na incisura. Por outro lado, corroboram dados que mostram que a atrofia e a MI são mais intensas na pequena curvatura antral ${ }^{(27)}$. Além do mais, na nossa casuística a presença de atrofia na pequena curvatura do antro médio estava significativamente associada com infecção por amostras cagA-positivas. A incisura foi a segunda área mais acometida e em alguns casos a MI foi mais acentuada nessa região. Assim, a população colombiana poderia representar um quadro avançado de GCAM e o envolvimento da incisura representaria a progressão da gastrite e não seu começo.

Em termos práticos, esse aspecto é importante porque é difícil mostrar a incisura à endoscopia e, provavelmente, isso não seria necessário para os diagnósticos corretos de GC e GCAM, uma vez que as lesões mais acentuadas são distais à incisura. A observação de que a porção média da pequena curvatura antral é uma área de risco para Ml está de acordo com o que se conhece em carcinogênese gástrica, uma vez que o $\mathrm{CaC}$ se desenvolve mais freqüentemente nessa área ${ }^{(4)}$.

Assim, concluindo, nossos dados sugerem que há diferentes padrões de gastrite associada a $H$. pylori relacionados a fatores de virulência da bactéria. Fatores do hospedeiro e ambientais devem modular o processo e sua evolução para a atrofia. As lesões pré-cancerosas são significativamente mais intensas na pequena curvatura do antro médio, onde surge a maioria dos $\mathrm{CaG}$.

\section{Referências}

I. BLASER, M.J. Infection with Helicobacter pylori strains possessing cagA is associated with an increased risk of developing adenocarcinoma of the stomach. Cancer Res, v. 55, p. 21 I I $5,1995$.

2. CLAYTON, C.L. et al. Sensitive detection of Helicobacter pylori by using polymerase chain reaction. J Clin Microbiol, v. 30, p. 192-200, 1992.

3. CORREA, P. Chronic gastritis: a clinico-pathological classification. Am J Gastroenterol, v. 83, p. 504-9, 1988.

4. CORREA, P. Helicobacter pylori and gastric carcinogenesis. Am J Surg Pathol, v. 19, s. I, p 37-43, 1995.

5. CORREA, P.; CUELLO, C.; DUQUE, E. Carcinoma and intestinal metaplasia of the stomach in Colombian migrants. I Nat Cancer Inst, v. 44, p. 297-306, 1970.

6. DIXON, M.F. et al. Classification and grading of gastritis: the Updated Sydney System. Am J Surg Pathol, v. 20, p. I|6|-8|, 1996.

7. DUNN, B.E.; COHEN, H.; BLASER, M.J. Helicobacter pylori. Clin Microbiol Ver, v. I0, p. 720-4I, 1997

8. EL-OMAR, E.M. et al. Helicobacter pylori infection and chronic gastric acid hyposecretion. Gastroenterology, v. I I3, n. I, p. I5-24, 1997.

9. EL-OMAR, E.M. et al. Interleukin-I polymorphism associated with increased risk of gastric cancer. Nature, v. 404, p 398402,2000
I0. FARINATI, F. et al. Helicobacter pylori CagA status, mucosal oxidative damage and gastritis phenotype: a potential pathway to cancer? Helicobacter, v. 8, p. 223-27, 2003.

I I. FIGUEIREDO, C. et al. Helicobacter pylori and interleukin I genotyping: an opportunity to identify high-risk individuals for gastric carcinoma. J Natl Cancer Inst, v. 94, p. 1680-7, 2002.

12. FILIPE, M.I. et al. Intestinal metaplasia types and the risk of gastric cancer: a cohort study in Slovenia. Int J Cancer, v .57, p. 324-9, 1994.

13. FORMAN, D. Is there significant variation in the risk of gastric cancer associated with Helicobacter pylori infection? Aliment Pharmacol Ther, v. 12, s. I, p. 3-7, 1998.

14. FUKUDA, S. et al. Helicobacter pylori infection, gastritis and gastric cancer. Histological analysis of gastritis and Helicobacter pylori infection in patients with early gastric cancer: a case-control study. J Gastroenterol Hepatol, v. I5, p. I370-6, 2000.

15. GENTA, R.M.; GÜRER, I.E.; GRAHAM, D.Y. Geographical pathology of Helicobacter pylori infection: is there more than one gastritis? Annals Med, v. 27, p. 595-9, 1995.

16. GENTA, R.M.; GRAHAM, D.Y. Comparison of biopsy sites for the histopathologic diagnosis of Helicobacter pylori infection: a topographic study of $\mathrm{H}$. pylori density and distribution. Gastrointest Endosc, v. 40, p. 342-5, 1994. 
17. HSU, S-M.; RAINE, L.; FANGER, H. Use of avidin-biotinperoxidase complex $(A B C)$ in immunoperoxidase techniques: a comparision between $\mathrm{ABC}$ and unlabeled antibody (PAP) procedures. J Histochem Cytochem, v. 29, p. $577-80,1981$.

18. KELLY, S.M. et al. Isolation of Helicobacter pylori from feces of patients with dyspepsia in the United Kingdom. Gastroenterology, v. 107, p. 1671-4, 1994.

19. KUIPPERS, E.J. et al. Helicobacter pylori and atrophic gastritis: importance of the cagA status. J Natl Cancer Inst, v. 87, p. 1777-80, 1995.

20. LAURÉN, P.The two histological main type of gastric carcinoma: diffuse and so-called intestinal type carcinoma; an attempt at a histoclinical classification. Acta Path et Microbiol Scandinav, v. 64, p. 31 - 49, 1965.

21. NOGUEIRA, C. et al. Helicobacter genotypes may determined gastric histopathology. Am J Pathol, v. I58, p. 647-54 2001.

22. PARSONNET,J. et al. Risk of gastric cancer in people with cagA positive or cagA negative Helicobacter pylori infection. Gut, v. 40, p. 297-30I, 1997.

23. PEEK JR., R.M. et al. Detection of Helicobacter pylori gene expression in human gastric mucosa. J Clin Microbiol, v. 33 p. 28-32, 1995.

24. QUEIROZ, D.M.M. et al. Cag-A positive Helicobacter pylori and risk for developing gastric carcinoma in Brazil. Int J Cancer, v. 78, p. 135-9, 1998

25. ROCHA, G.A. et al. Simple carbolfuchsin staining for showing C pylori and other spiral bacteria in gastric mucosa. J Clin Pathol, v. 42, p. 1004-5, 1989.

26. RUGGE, M. et al. Pathology of the gastric antrum and body associated with Helicobacter pylori infection in non-ulcerous patients: is the bacterium a promoter of intestinal metaplasia? Histopathology, v .22, p. 9-15, 1993.

27. SATOH, K. et al. Biopsy sites suitable for the diagnosis of Helicobacter pylori infection in the assessment of the extent of atrophic gastritis. Am J Gastroenterol, v. 93, p. 569-73, 1998.

28. SCHOLTE, G.H. et al. Genotyping of Helicobacter pylori strains in formalin-fixed or formaldehyde-sublimate-fixed paraffinembedded gastric biopsy specimens. Diagn Mol Pathol, v. 10 , p. $|66-70,200|$

29. SOZZI, M. et al. Atrophic gastritis and intestinal metaplasia in Helicobacter pylori infection: the role of cagA status. Am J Gastroenterol, v. 93, p 375-9, 1998.

30. SPICER S.S. Diamine methods for differentiating mucosubstances histochemically. J Histochem Cytochem; v. I 3, p. 2 I I-34, 1965.

31.WARBURTONV.V.J.J et al. Clinical and histological associations of cagA and vacA genotypes in Helicobacter pylori gastritis. J Clin Pathol, v. 5I, p. 55-6I, 1998. 